\title{
The Study of Vibration Phenomenon of Hard-Disk Disk
}

\author{
Dong-Sheng Lin ${ }^{12, a}$ and Yi-Jui Chiu ${ }^{12, b}$ \\ ${ }^{1}$ School of Mechanical and Automotive Engineering, Xiamen University of Technology, No. 600, \\ Ligong Rd, Xiamen, 361024,Fujian Province, China. \\ ${ }^{2}$ Collaborative innovation Center for R\&D Coach and Special Vehicle of Fujian Province. \\ a892171397@qq.com, bchiuyijui@xmut.edu.cn
}

Keywords: hard-disk disk, vibration.

Abstract. The intention of this paper is to explore the dynamic behavior of hard-disk disk. In this paper, the modal analysis of hard-disk disk simulated by ANSYS and the vibration and the resonance behavior are introduced .The results of this research could provide engineers with useful information to understand the dynamic vibration behavior and design the frame of hard disk.

\section{Introduction}

The data disk is an important part of a hard disk with which covered a ferromagnetic material. It affects the reliability and longevity of the hard disk. Therefore, the modal parameters of data disks are important factors in hard disk design. Modal analysis is a procedure to determine the vibration characteristics of structure and gain natural frequency. Some researches, such as Yap et. al. [1] reviewed recent advances in shock and vibration analysis of hard disk drives considering the presence of nonlinearities and discontinuities, and they applied a half-sinusoidal acceleration shock at the base of the HDD. Nayfeh et.al.[2] used the multiple scales method, and determined a set of four nonlinear ordinary-differential equations governing the modulation of the amplitudes and phases of two interacting modes. Lee et.al.[3] utilized a vacuum chamber and optical disks give two main results. One is that the aerodynamic effect by surrounding air reduces the natural frequencies and critical speeds of the vibration modes in pre-flutter regions. The other is that the natural frequency of the disk rotating at ambient atmospheric pressure is equal to that in vacuum at the flutter onset speed where the disk experiences aero-induced flutter.

This paper took virtue 3D models of hard disk's main frames made by Pro/Engineer. Hard disk models are shown in Fig.1. This paper calculated the free vibrations hard disk frameworks, and obtained the natural frequencies and vibration modes of the frame structure by using ANSYS. Second, the paper intended to provide a qualitative and quantitative overview of hard-disk disk.

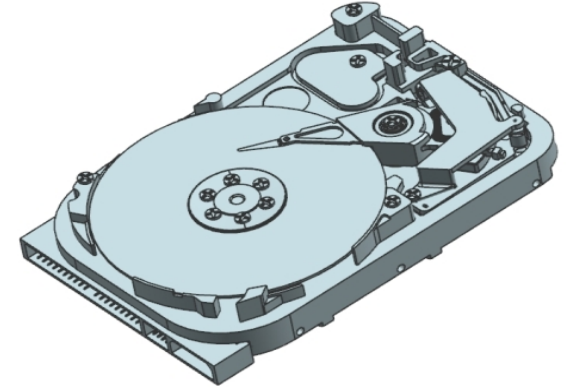

(a)

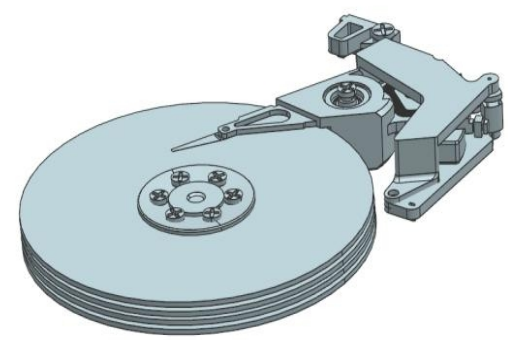

(b)

Fig. 1. 3D models of hard-disk disk

\section{Dynamic Analysis}

Because the structure of main frame was very complex, we should simplify the structure first. $[\mathrm{M}]\{X\}+[\mathrm{K}]\{X\}=0$

Defined the position vector $\{X\}$ as $[\Delta]\{u\}$, where $[\Delta]$ was the modal matrix of the system. Eq. 
(2) could be changed as follow:

$$
[I]\{+[A]\{u\}=0
$$

In which:

$$
\begin{gathered}
{[\Delta]^{T}[M][\Delta]=[I]=\left[\begin{array}{cccc}
1 & 0 & \mathrm{~L} & 0 \\
0 & 1 & \mathrm{~L} & \mathrm{M} \\
\mathrm{M} & 0 & \mathrm{O} & 0 \\
0 & 0 & 0 & 1
\end{array}\right]} \\
{[\Delta]^{T}[K][\Delta]=[A]=\left[\begin{array}{cccc}
{\overline{\omega_{1}}}^{2} & 0 & \mathrm{~L} & 0 \\
0 & \bar{\omega}_{2}^{2} & \mathrm{~L} & \mathrm{M} \\
\mathrm{M} & 0 & 0 & 0 \\
0 & \mathrm{~L} & 0 & {\frac{\omega_{n}}{2}}^{2}
\end{array}\right]}
\end{gathered}
$$

The natural frequency of the mistuned system was expressed as follow:

$$
\overline{\omega_{n}}=\frac{\omega_{n}}{\sqrt{\frac{E I}{\rho A L^{4}}}}, n=1,2,3 \ldots \ldots
$$

\section{Numerical Results and Analysis}

The sizes of main frame were given in Table 1. And aluminum is the material we mainly used for the main frame. Because of the premise of finite element analysis, we tried to simplify the geometric model of the main frames and took aluminum as the main material to obtain the main structure characteristics . The physical parameters of material aluminum is shown in Table 2.

Table 1.The Main Frame Sizes

\begin{tabular}{|c|c|c|c|}
\hline Type & $\begin{array}{c}\text { Outer } \\
\text { diameter }(\mathrm{mm})\end{array}$ & $\begin{array}{c}\text { Inner } \\
\text { diameter }(\mathrm{mm})\end{array}$ & Thickness $(\mathrm{mm})$ \\
\hline Hard disk & 118 & 36 & 2 \\
\hline
\end{tabular}

Table 2. The Physical Parameters of Material aluminum

\begin{tabular}{|c|c|}
\hline Project & Value \\
\hline Yield Point & $276 \mathrm{Mpa}$ \\
\hline Young's Modulus & $69 \mathrm{Gpa}$ \\
\hline Poisson's Ratio & 0.3 \\
\hline Density & $2.73 \mathrm{~kg} / \mathrm{m}^{3}$ \\
\hline
\end{tabular}

The element type we used is Solid 186. Because Solid 186 is a high order 3D structure unit with 20 nodes, since it has a pattern of two times displacement, which means it can simulate irregular meshes in a better way, Simulation can be more accurate with Solid 186. In addition, there is no restraint for the body frame. First, we used ANSYS that get the data results are shown in Table 3. In this Table, we found some models frequencies are repeated frequencies. 
Table 3. Natural Frequencies (Hz) of hard-disk disk, only used ANSYS

\begin{tabular}{|c|c|c|c|c|c|}
\hline Modes & 1 & 2 & 3 & 4 & 5 \\
\hline Frequencies $(\mathrm{Hz})$ & 1171.74 & 1171.74 & 1197.46 & 1197.46 & 1206.32 \\
\hline Modes & 6 & 7 & 8 & $9^{1}$ & 10 \\
\hline frequencies $(\mathrm{Hz})$ & 1210.82 & 1210.82 & 1210.82 & 1210.82 & 1218.21 \\
\hline Modes & 11 & 12 & 13 & 14 & 15 \\
\hline Frequencies $(\mathrm{Hz})$ & 1224.13 & 1224.13 & 1379.63 & 1379.63 & 1434.15 \\
\hline Modes & 16 & 17 & 18 & 19 & 20 \\
\hline frequencies $(\mathrm{Hz})$ & 1434.15 & 1468.33 & 1468.33 & 1468.33 & 1468.33 \\
\hline Modes & 21 & 22 & 23 & 24 & 25 \\
\hline Frequencies $(\mathrm{Hz})$ & 2373.94 & 2373.94 & 2411.2 & 2411.2 & 2446.31 \\
\hline Modes & 26 & 27 & 28 & & \\
\hline frequencies $(\mathrm{Hz})$ & 2446.31 & 2446.31 & 2446.31 & & \\
\hline
\end{tabular}

We used the linear elastic thin plate theory [4] and check the models deformation. We observe new results are shown in Table 4. The modes of hard disk are shown in Fig. 2 to Fig. 5. In this paper, we only show some modes, not all.

Table 4. Natural Frequencies of hard-disk disk, used linear elastic thin plate theory

\begin{tabular}{|c|c|c|c|c|c|}
\hline Modes & $(0,1) \mathrm{a}$ & $(0,1) \mathrm{b}$ & $(0,0) \mathrm{a}$ & $(0,1) \mathrm{c}$ & $(0,0) \mathrm{b}$ \\
\hline Frequencies $(\mathrm{Hz})$ & 1171.74 & 1197.46 & 1206.32 & 1210.82 & 1218.21 \\
\hline Modes & $(0,0) \mathrm{c}$ & $(0,2) \mathrm{a}$ & $(0,2) \mathrm{b}$ & $(0,2) \mathrm{c}$ & $(0,3) \mathrm{a}$ \\
\hline frequencies $(\mathrm{Hz})$ & 1224.13 & 1379.63 & 1434.15 & 1468.33 & 2373.94 \\
\hline Modes & $(0,3) \mathrm{b}$ & $(0,3) \mathrm{c}$ & & & \\
\hline Frequencies $(\mathrm{Hz})$ & 2411.2 & 2446.31 & & & \\
\hline
\end{tabular}

From Table 4 and Fig. 2 to Fig. 5, this paper found some interesting results as follow. First, the modes behavior sequence are $(0,1),(0,0),(0,2)$ and $(0,3)$, the results follow closely with the linear elastic thin plate theory. Second, $(0,1),(0,2)$ and $(0,3)$ modes have three groups modes. Such as $(0,1)$ modes, $(0,1) \mathrm{a}$ and $(0,1) \mathrm{b}$ modes are inner disks modes were of repeated frequencies of $N_{d} / 2$ multiplicity for number disks. The $(0,1) \mathrm{c}$ modes are outer disks modes were of repeated frequencies of $N_{d}$ multiplicity for number disks. The phenomenon are similar as $(0,2)$ and $(0,3)$ modes. Third, $(0,0)$ modes have three groups modes. But, $(0,0) \mathrm{a}$ and $(0,0) \mathrm{b}$ modes are inner disks modes only has a single frequency. The $(0,0) \mathrm{c}$ modes are outer disks modes were of repeated frequencies of $N_{d} / 2$ multiplicity for number disks.

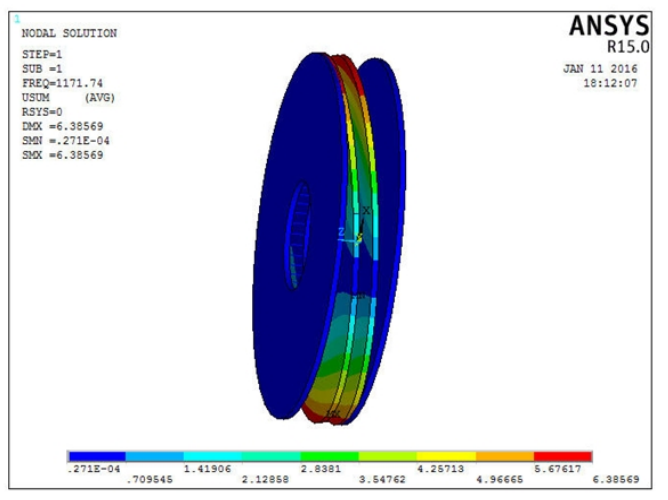

(a) $(0,1)$ a

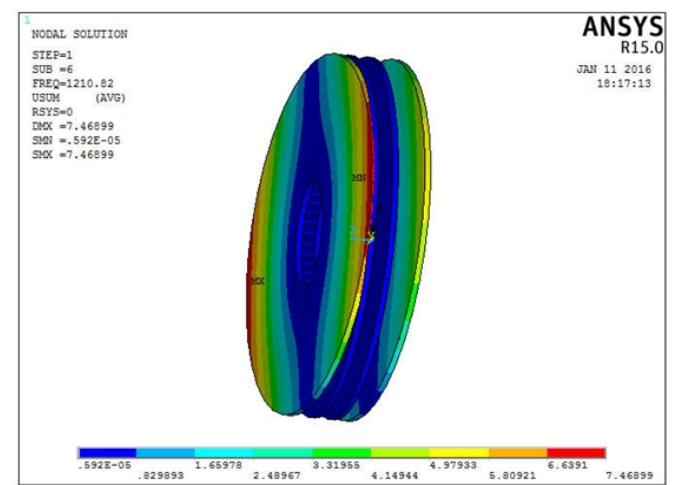

(b) $(0,1) \mathrm{c}$

Fig. 2. The $(0,1)$ modes of hard-disk disk 


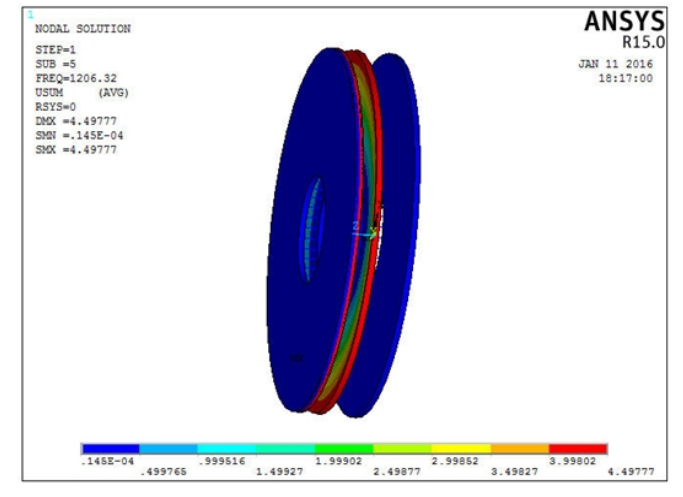

(a) $(0,0)$ a

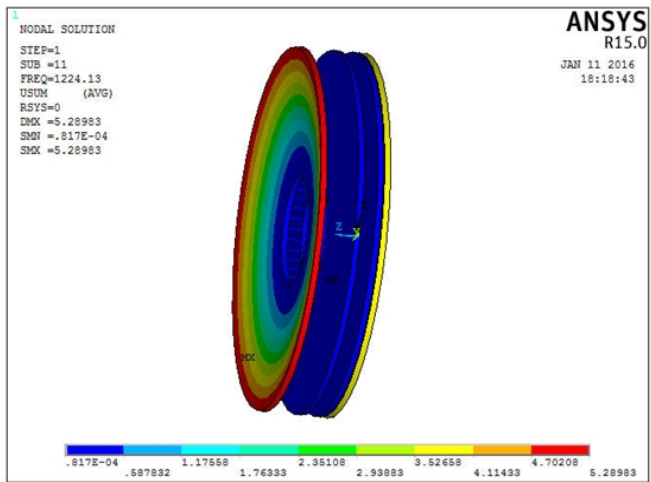

(b) $(0,0) \mathrm{c}$

Fig. 3. The $(0,0)$ modes of hard-disk disk

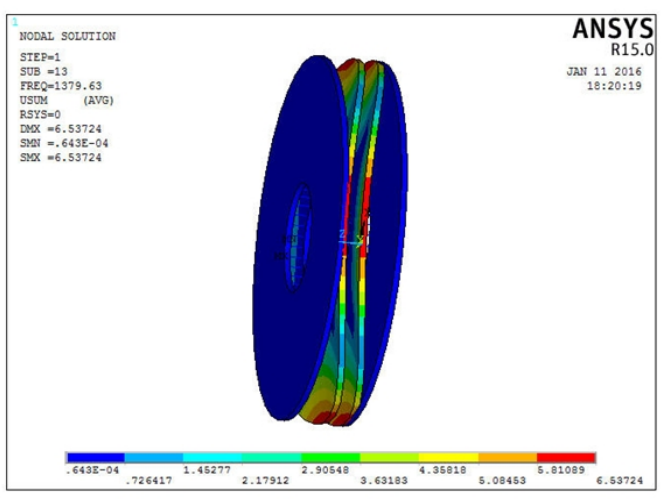

(a) $(0,2) \mathrm{a}$

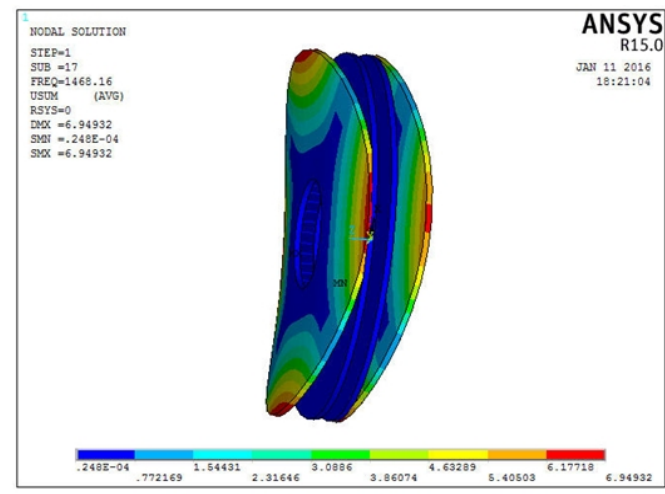

(b) $(0,2) \mathrm{c}$

Fig.4. The $(0,2)$ modes of hard-disk disk

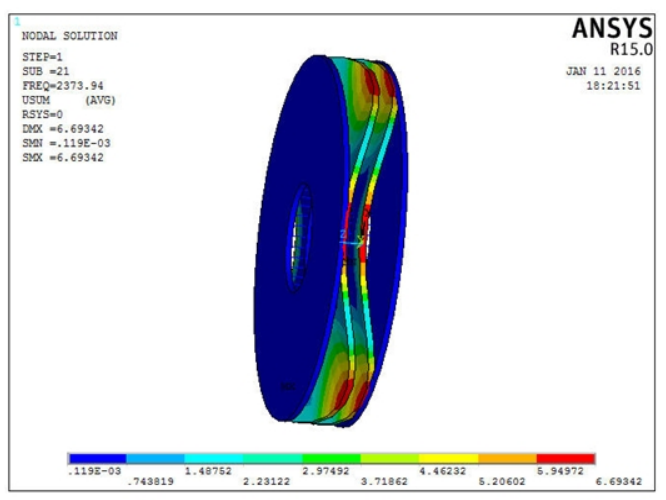

(a) $(0,3)$ a

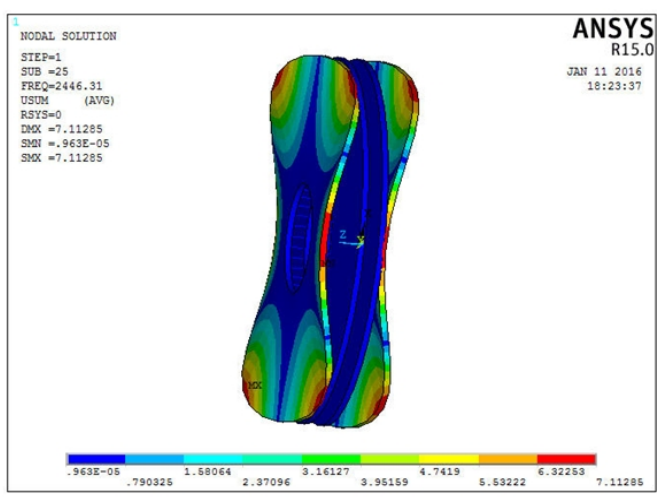

(b) $(0,3) \mathrm{c}$

Fig. 5. The $(0,3)$ modes of hard-disk disk

\section{Conclusion}

This paper used the ANSYS that is commercial software to perform finite element analysis. The cases of a qualitative and quantitative overview of hard-disk disk have been explored in this paper. The results show three interesting results. First, the results follow closely with the linear elastic thin plate theory. Second, $(0,1),(0,2)$ and $(0,3)$ modes of inner disks modes were of repeated frequencies of $\mathrm{N}_{\mathrm{d}} / 2$ multiplicity for number disks. The outer disks modes were of repeated 
frequencies of $\mathrm{Nd}$ multiplicity for number disks. Third, $(0,0)$ modes of inner disks modes only has a single frequency. The outer disks modes were of repeated frequencies of $\mathrm{N}_{\mathrm{d}} / 2$ multiplicity for number disks.

\section{Acknowledgement}

This work was financially supported by the Fujian Nature foundation (2016J01039). Fujian Department of education foundation (JAS150434). Qiantang river talent foundation (2013R10075). Zhejiang Nature foundation (Y14A020001). Xiamen University of Technology talent foundation (YKJ15027R). National Natural Science Foundation of China (51475399).

\section{References}

[1] F.F. Yap, H. Harmoko, M. Liu and N. Vahdati, Nonlinear Dynamics, Vol. 50 (2007), p.717-731. [2] A. Nayfeh, A. Jilani and P. Manzione, Nonlinear Dynamics, Vol. 26 (2001), p. 163-178.

[3] S.Y. Lee, D.W. Yoon and K. Park, Microsystem Technologies, Vol. 9 (2003), p. 369-374.

[4] Y.J. Chiu and S.C. Huang, Shock and Vibration, Vol. 15(1) (2008), p. 3-17. 